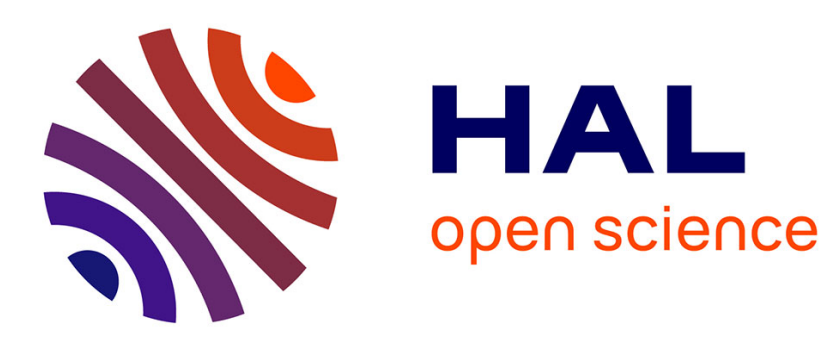

\title{
Use of Wollaston prism for dual-reference digital holographic interferometry
}

\author{
Jean-Michel Desse, François Olchewsky
}

\section{To cite this version:}

Jean-Michel Desse, François Olchewsky. Use of Wollaston prism for dual-reference digital holographic interferometry. Digital Holography and 3D Imaging, May 2019, BORDEAUX, France. 10.1364/DH.2019.Tu4B.4 . hal-02301557

\section{HAL Id: hal-02301557 \\ https://hal.science/hal-02301557}

Submitted on 30 Sep 2019

HAL is a multi-disciplinary open access archive for the deposit and dissemination of scientific research documents, whether they are published or not. The documents may come from teaching and research institutions in France or abroad, or from public or private research centers.
L'archive ouverte pluridisciplinaire HAL, est destinée au dépôt et à la diffusion de documents scientifiques de niveau recherche, publiés ou non, émanant des établissements d'enseignement et de recherche français ou étrangers, des laboratoires publics ou privés. 


\title{
Use of Wollaston prism for dual-reference digital holographic interferometry
}

\author{
Jean-Michel Desse $e^{1,2 *}$, François Olchewsky ${ }^{1,2}$ \\ IONERA, DAAA, 5, rue des Fortifications, CS 90013, F-59045 LILLE, France \\ ${ }^{2}$ Laboratoire de Mécanique des Fluides de Lille - Kampé de Fériet, F-59000 LILLE France \\ *Jean-Michel.Desse@onera.fr
}

\begin{abstract}
A Wollaston prism is used in digital holographic interferometer developed for analyzing high density gradients encountered in transonic and supersonic flows. Inserted in the reference arm of the interferometer, the Wollaston prism allows generating two orthogonally crossed waves and analyzing shocks waves whatever their orientation. OCIS codes: 090.0090, 090.2880, 090.1995.
\end{abstract}

\section{Introduction}

As part of the work carried out on the development of digital holographic interferometry by ONERA, the authors propose a new double-reference digital holographic interferometer for the analysis of the strong variations of refractive index encountered, for example, in transonic and supersonic flows. Recent studies have shown the feasibility of a Mach-Zehnder digital holographic interferometer for the analysis of subsonic high flows [1]. Conclusive results were obtained on the unsteady flow downstream of a circular cylinder located transversely in the test section of experiments. But when the Mach number reaches transonic domain, the vortices emitted from the upper and lower side of the cylinder generate shock waves which spread everywhere in the field. As consequence, the grating of interference fringes generated in the field may be parallel to the orientation of the shock waves, which causes residual singularities during the unwrapping operation of modulo $2 \pi$ phase maps. To circumvent this inevitable problem, the authors developed a double-reference digital holographic interferometer by inserting a Wollaston prism into the reference arm of the interferometer to simultaneously generate two orthogonally polarized reference waves and thus, the recorded interferograms contain two crossed and perpendicular interference patterns that give two completely separate orders in the Fourier spectrum. It is then possible to analyze a transparent object whatever the orientation of the refractive index gradient by using the two reconstructed phase maps with each of the first two orders of interference. The fusion of the 2 phase maps gives a single phase map in which the phase singularities are eliminated. The experimental results demonstrate the relevance of the proposed approach for the analysis of shock waves in the unsteady wake at Mach 0.75.

\section{Experimental setup and Result}

The optical scheme shown in Fig.1 represents a Mach-Zehnder interferometer. A pulsed laser (5 to 6 nanoseconds) of about 3 meters in coherence length is used as luminous source. A beam splitter cube forms the object beam that passes through the test section and the reference beam that passes below. Object wave side, a spatial filter associated with the f $800 \mathrm{~mm}$ lens allows illuminating the test section with a parallel beam. A second f800mm lens associated with the $f 70 \mathrm{~mm}$ lens fixs the magnification of the observed field onto the camera sensor. Reference wave side, a second spatial filter and a f120mm lens form a parallel beam on the plane mirror. A last beam splitter cube recombines the parallel beams (reference and object) on the sensor. On the reference arm, a Wollaston prism is inserted just behind the spatial filter. It separates the reference wave into two polarized waves orthogonally $\mathrm{S}$ and $\mathrm{P}$ (red and blue). These two waves are separated by a birefringence angle that depends on the pasting angle of the prism and the prism material (quartz or calcite). Given the focal length of the lens $(120 \mathrm{~mm})$, the birefringence angle was calculated to obtain on the sensor a fringe space of the order of ten pixels. This requires the use of a Wollaston prism in calcite with a pasting angle of $8^{\circ}$.

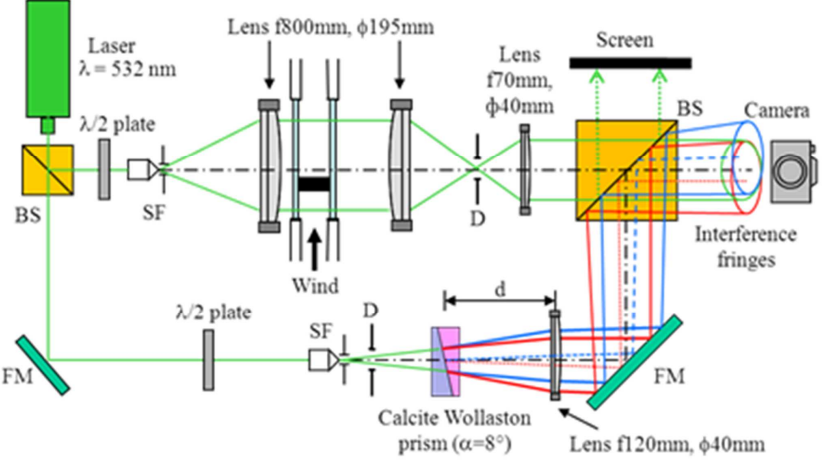

Fig. 1 Dual-reference digital holographic interferometer 
Figure 2 shows how to generate two patterns of interference fringes inclined to the left and right. On reference side, the two blue and red waves are separated by a distance $\mathrm{dx}$ that depends on the birefringence angle of the Wollaston prism. The blue beam has a polarization $\mathrm{P}$ and the red beam $\mathrm{a}$ polarization S. On the horizontal axis, the object wave is arranged between the two reference waves at the distance $\mathrm{dx} / 2$ and on the vertical axis, the reference beam is placed at a distance equal to $\mathrm{dx} / 2$. Thus, for $\mathrm{P}$ polarized beams (blue and green), interference fringes are generated at $+45^{\circ}$ and for $\mathrm{S}$ polarized beams, interference fringes are obtained at $-45^{\circ}$. These two patterns are superimposed on the sensor and the recording window is placed in the common part of the two interferences.

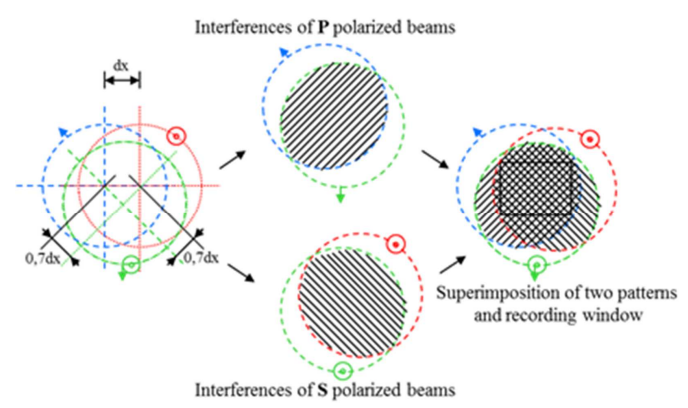

Fig. 2 Oblique and orthogonal fringes

Then, the interferences of the reference hologram are recorded in the absence of the phenomenon to be studied (without flow), then with flow (Mach 0.75). The Fourier spectra of the reference and measurement images are calculated for the two polarization states. Spectral filtering is performed to keep only the information of interest (order +1 ) and the phase and phase difference maps between the reference and the measurement for the two polarization states are computed. Figure 3 shows the unwrapped phase maps for the 2 polarization states. It can be seen that there are already phase dislocations that cannot be resolved (surrounded by white ellipses). These dislocations appear only in the upper part of the polarized image $S$ and in the lower part of the polarized image $\mathrm{P}$. We will thus be able to analyze the upper part by taking the interferogram coming from the polarizer $\mathrm{P}$ and analyze the lower part by taking the interferogram provided by the polarization $S$. Fig. 4 shows the result of this combination analysis. From a point of view of fluids mechanics, a phase variation $\Delta \phi$ of about 120 radians between the stagnation point (just at the nose of the cylinder) and the

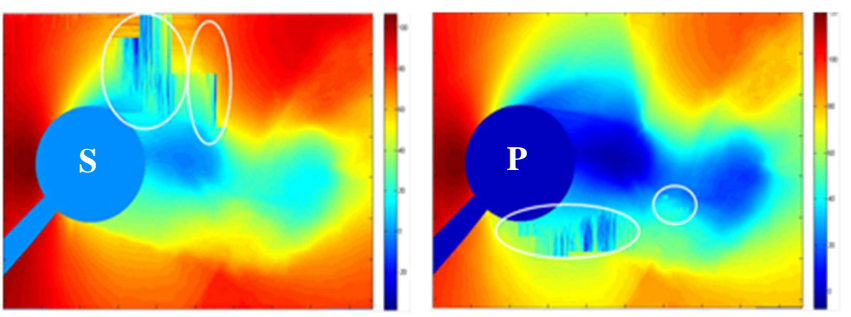

Fig. 3 Unwrapped phase map $-\mathrm{S}$ and $\mathrm{P}$ polarizations

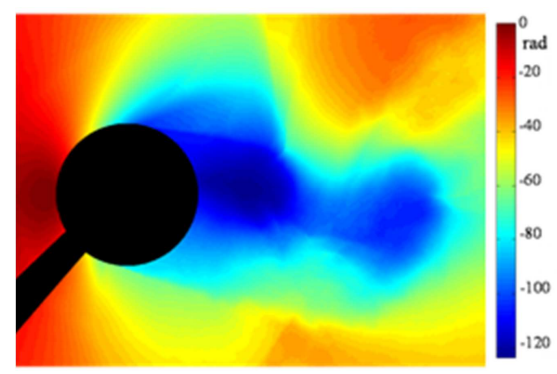

Fig. 4 Result of fusion center of the first vortex downstream the cylinder can be measured from the experimental results. It follows that the gas density in the first vortex exhibits a strong decrease down to $90 \%$ of the stagnation gas density.

\section{Conclusion}

In conclusion, a dual-reference digital holography using a Wollaston prism is presented for analyzing high density gradients in fluids mechanics. As consequence, two reference waves orthogonally polarized are simultaneously generated. The recorded interferogram contains two crossed perpendicular interference patterns which give two signatures fully separated in 2DFFT spectrum. It is then possible to analyze a transparent object regardless the orientation of refractive index gradient using the two phase maps reconstructed with each of the two first orders of interference. This interferometer is applied for analyzing shock waves in the unsteady wake flow around a circular cylinder at Mach 0.75 .

\section{Acknowledgment}

This research was partially funded by the French National Agency for Research (ANR) under grant agreement $n^{\circ}$ ANR 2010 BLAN 0302.

\section{Reference}

[1] Desse J.M. et al., "Digital color holography for analyzing unsteady wake flows" in New Techniques in Digital Holography, E.P. Picart, ed. (Iste-Wiley, Hermes-science publishing, 2015). 